\title{
Index for Fast Retrieval of Uncertain Spatial Point Data .
}

\author{
Dmitri V. Kalashnikov Yiming Ma Sharad Mehrotra Ramaswamy Hariharan \\ Information and Computer Science \\ University of California, Irvine, USA
}

\begin{abstract}
Location information gathered from a variety of sources in the form of sensor data, video streams, human observations, and so on, is often imprecise and uncertain and needs to be represented approximately. To represent such uncertain location information, the use of a probabilistic model that captures the imprecise location as a probability density function (pdf) has been recently proposed. The pdfs can be arbitrarily complex depending on the type of application and the source of imprecision. Hence, efficiently representing, storing and querying pdfs is a very challenging task. While the current state of the art indexing approaches treat the representation and storage of pdfs as a black box, in this paper, we take the challenge of representing and storing any complex pdf in an efficient way. We further develop techniques to index such pdfs to support the efficient processing of location queries. Our extensive experiments demonstrate that our indexing techniques significantly outperform the best existing solutions.
\end{abstract}

\section{Categories and Subject Descriptors}

H.2.8 [Information System]: Database ManagementSpatial databases and GIS

\section{General Terms}

Algorithms, Performance

\section{Keywords}

Spatial Database, Uncertainty Indexing, U-grid, Probability

\section{INTRODUCTION}

With the emergence of mobile computing and ubiquitous connectivity, numerous new applications that exploit location information have emerged (e.g, location based search,

${ }^{*}$ This research was sponsored by NSF Award number 0331707 and 0331690 to the RESCUE project [12].

Permission to make digital or hard copies of all or part of this work for personal or classroom use is granted without fee provided that copies are not made or distributed for profit or commercial advantage and that copies bear this notice and the full citation on the first page. To copy otherwise, to republish, to post on servers or to redistribute to lists, requires prior specific permission and/or a fee.

ACM-GIS'06, November 10-11, 2006, Arlington, Virginia, USA

Copyright 2006 ACM 1-59593-529-0/06/0011 ...\$5.00. location based services, etc.) In such applications, location information of individuals (or devices) is determined in various ways. These include location sensors such as GPS, WiFi access point triangulation, cell phones, etc. Location information may also be derived from conversations wherein people describe their location through speech and/or text.

In all of these cases, location information is often imprecise. Devices such as GPS, cell phones, etc. have fundamental limitation on their localization capabilities. When people report their locations or describe events, they usually do so in an imprecise manner - e.g., "an accident happened on Freeway 405 near McArthur Street".

Developing location-based systems requires mechanisms to represent, query and reason with location uncertainty. A model for representing location uncertainty that has recently emerged is the probabilistic spatial model $[2,3,6]$. In such a model, uncertain location is represented as a probability of the event/object to have occurred at a given location (i.e., a probability density function over the spatial domain). Such a probabilistic model is powerful for a variety of reasons. First, it can be used to represent not only simple cases of uncertainty that arise due to device errors, but also fairly complex types of uncertainties that may arise in interpreting textual descriptions of location and many other applications [15]. Another advantage of the probabilistic model is that it allows for a clear and formal interpretation of spatial queries and has been demonstrated to be effective in practice.

While probabilistic model provides a powerful framework for representing uncertain location data, it introduces new challenges in supporting spatial queries efficiently. In this paper, we develop an efficient approach to indexing uncertain location information represented using probabilistic distributions over space. We develop efficient query processing algorithms that exploit the index structure developed for efficient retrieval. Specifically, we focus on probabilistic range query (RQ) and preference range query (PRQ), which are described later. The main contributions of this paper are:

- A novel indexing framework and algorithms for efficient spatial query processing (Sections 5 and 6).

- Extensive empirical evaluation of the proposed approach (Section 7).

The rest of the paper is organized as follows. Section 2 covers the related work. Section 3 defines two types of probabilistic retrieval queries. We then discuss ways to represent uncertain locations in a database in Section 4. Sections 5 and 6 introduce the novel index, U-grid, and query processing algorithms. In Section 7 we empirically evaluate the proposed approach. Finally, we conclude in Section 8. 


\section{RELATED WORK}

In this section, we review most related work on representing spatial uncertainty, indexing, and query processing.

Uncertain location data representation. We will use the probabilistic model for spatial uncertainty developed in $[3,5-8]$, because it builds on the formal probability theory and it has been shown to be very effective in practice. Similar models are also employed in $[2,4]$. In the probabilistic model, an uncertain location $\ell$ is treated as a continuous random variable (r.v.) which takes values $(x, y)$ inside domain $\Omega$ and has a certain probability density function (pdf) $f_{\ell}(x, y)$ associated with it. Interpreted this way, for any spatial region $R$, the probability that $\ell$ is inside $R$ is computed as $\int_{R} f_{\ell}(x, y) d x d y$.

Representation. In many applications that deal with uncertain event locations [8,11], we need to be able to represent pdfs of complex shapes in the database. There are several known methods for such a representation, such as histograms and modeling pdf as a mixture of Gaussians or of other distributions. In our approach, we will represent pdfs as histograms, which are stored in the database as quad-trees. Using quad-trees allows us to achieve fast query response time and also to compress those histograms (Section 4). It is interesting to note that the existing solutions that also deal with probabilistic spatial queries $[3,6]$ do not address the pdf representation issues directly. The reason is that their empirical evaluation is carried out using only simple densities such as uniform and Gaussian.

Indexing and query processing. In Sections 5 and 6 we introduce novel indexing and query processing techniques. Indexing pdfs to support efficient query processing has been explored in previous literature as well $[3,6]$. A typical approach is to restrict possible values of an uncertain location $\ell$ to be inside an uncertainty region $U_{\ell}$ such that $f_{\ell}(x, y)=0$ if $(x, y) \notin U_{\ell}$. Figure 1 (a) in Section 3 illustrates that concept by showing that locations of events $a_{1}, a_{2}, a_{3}, a_{4}$ are restricted to their respective uncertainty regions (shaded). The uncertain location is indexed using data structures such as R-tree based on their uncertainty region. This allows answering certain spatial queries without performing costly integration operations. For instance, by analyzing the uncertainty regions in Figure 1(a) it is clear that the location of $a_{1}$ is guaranteed to be inside the range $R$.

Current state of the art. The $x$-bounds and U-tree are the current state of the art techniques studied in $[6,15]$ for 1and $n$-dimensional cases. Both techniques have been implemented as variations of R-tree. For 1-dimensional case, the idea is to store in each internal node $\mathcal{N}$ of an $\mathrm{R}$-tree extra information: left and right ' $x$-bounds' for several values of $x$, e.g. $x=0.1$ and $x=0.2$. A 'left $x$-bound' of $\mathcal{N}$ is any value $l(x) \in \mathbb{R}$ such that for any pdf $f(z)$ covered by $\mathcal{N}$ it follows that $\int_{-\infty}^{l(x)} f(z) d z<x$. A 'right $x$-bound' is defined similarly. So, if a probabilistic threshold query (see Sec. 3) with range $R$ and threshold $p_{\tau}$ overlaps the MBR of $\mathcal{N}$, but $\mathcal{N}$ stores a left $x$-bound such that (1) $R \subset(-\infty, l(x)]$; and (2) $p_{\tau}>x$, then $\mathcal{N}$ is pruned since it cannot contain any object (pdf) that would satisfy the $\tau$-RQ. The idea of $x$-bounds generalizes to 2-dimensional case, but now, in addition to left and right $x$-bounds, top and bottom $y$-bounds should be maintained as well. Keeping $x$-bounds can increase the height of the R-tree due to the extra information kept in nodes of the R-tree, negatively affecting performance. Cheng et

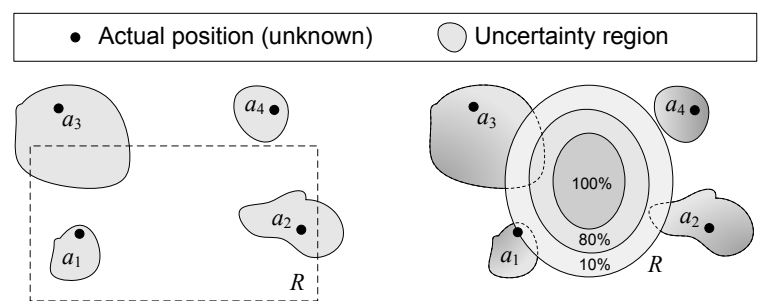

(a) RQ with range $R$

(b) PRQ with pref. region $\mathrm{R}$

Figure 1: Examples of $\mathbf{R Q}(R)$ and $\operatorname{PRQ}(q)$.

al. show in [6] that the gains of $x$-bounds outweigh their disadvantages in 1-dimensional case. Notice, unlike traditional methods, the $x$-bound approach employs for pruning not only spatial part (i.e. MBR), but also values of the pdf.

\section{SPATIAL QUERIES}

In this section, we formally define two types of probabilistic retrieval queries studied in this paper. Similar queries have been considered in [2,3]. The first fundamental type of query is a range or region query, such as "find all the events, the location of which can be inside a given region". Those queries can be formally defined as:

Definition 1. Given a region (range) $R$ and a set of objects $A=\left\{a_{1}, a_{2}, \ldots, a_{n}\right\}$, a basic region (range) query (RQ) returns all the elements in A whose probability of being inside $R$ is greater than zero.

The analytical formula for computing the probability that a location $\ell \sim f_{\ell}(x, y)$ is located inside a region $R$ is given by $\mathrm{P}(\ell \in R)=\int_{R} f_{\ell}(x, y) d x d y$.

Consider the example in Figure 1(a). If we assume the locations of elements $A=\left\{a_{1}, a_{2}, a_{3}, a_{4}\right\}$ are uniformly distributed in the specified (shaded) uncertainty regions, then the probabilities of these elements of being inside $R$ might be $1.0,0.7,0.4,0.0$ respectively. Then the result set for the corresponding RQ is $\left\{a_{1}, a_{2}, a_{3}\right\}$.

Having only elements in the result set might not be always sufficient: it is often necessary to be able to get the actual values of the probabilities associated with those elements, which leads us to the next definition:

Definition 2. A probabilistic query is a detached-probability query if it returns elements without the probabilities associated with them. A query is an attached-probability query, denoted as $p$-, if its result is a set of tuples where each tuple consists of an element and the probability associated with this element.

By default, any spatial query is a detached-probability query. The answer set for the $p$-RQ counterpart of the above $\mathrm{RQ}$ is $\left\{\left\langle a_{1}, p_{1}\right\rangle,\left\langle a_{2}, p_{2}\right\rangle,\left\langle a_{3}, p_{3}\right\rangle\right\}$. Given that we have assumed $p_{1}, p_{2}, p_{3}$ have specific values, the answer is $\left\{\left\langle a_{1}, 1.0\right\rangle\right.$, $\left.\left\langle a_{2}, 0.7\right\rangle,\left\langle a_{3}, 0.4\right\rangle\right\}$.

When analyzing results of an RQ, it is often desirable to ignore low-probability answers and present only those elements whose associated probabilities exceed a given probability threshold $p_{\tau}$, where $p_{\tau} \in \mathbb{R}, 0 \leq p_{\tau} \leq 1$. To generalize this idea, we can define:

DeFinition 3. Given a threshold $p_{\tau}$, query $\tau-Q$ is said to be query $Q$ with the threshold semantics if on the same input as $Q$ it returns all the elements from the result set of $Q$ whose associated probabilities are greater than $p_{\tau}$. 
To continue with our running example, if we set $p_{\tau}=0.5$, then the corresponding $\tau$-RQ will return $\left\{a_{1}, a_{2}\right\}$ as its result set, because $p_{1}$ and $p_{2}$ are greater than 0.5 whereas $p_{3}$ and $p_{4}$ are not. Similarly, $p \tau$-RQ will return $\left\{\left\langle a_{1}, 1.0\right\rangle,\left\langle a_{2}, 0.7\right\rangle\right\}$.

Notice, we have deliberately defined each $\tau$-RQ as a single operation, and not as an additional filtering ( $\tau$ - part) step applied to RQ operation. This is because $\tau$-RQ, as a single query, can be optimized better, which is important for quick query response time.

Finally, observe that a regular region $R$ can be viewed as a discrete function $R(x, y): \Omega \rightarrow\{0,1\}$, such that $R(x, y)=$ 1 when $(x, y) \in R$, and $R(x, y)=0$ otherwise. The formula for $\mathrm{P}(\ell \in R)$ can be written as

$$
\mathrm{P}(\ell \in R)=\int_{\Omega} f_{\ell}(x, y) R(x, y) d x d y .
$$

The concept of a regular region generalizes to the concept of a preference region $R(x, y): \Omega \rightarrow[0,1]$, which maps a point to a preference value between zero and one. Similarly, the concept a region query generalizes to the concept of a preference region query (PRQ): everything is the same as for $\mathrm{RQ}$, except $\mathrm{P}(\ell \in R)$ is computed using Equation 1. PRQs give the flexibility to specify queries where, for example, the analyst is primarily interested in objects that are within a certain area of space, but also cannot ignore object in a larger area of space. An example of a PRQ with the preference region $R$ is illustrated in Figure 1(b). There, the analyst prefers objects in the inner oval $(R(x, y)=1)$ to object in the middle ring $(R(x, y)=0.8)$ to objects in the outer ring $(R(x, y)=0.1)$. More complex preference regions can be defined, e.g. $R(x, y)$ can be a continuous function.

\section{REPRESENTING PDFS}

\subsection{Histogram representation of pdfs}

In order to represent and manipulate pdfs with complex shapes, we first quantize the space by viewing the domain $\Omega$ as a fine uniform grid $G$ with cells of size $\delta \times \delta$. The grid $G$ is virtual and is never materialized. We use the same notation $G_{i j}$ for both the cell in $i$-th row and $j$-th column of $G$, i.e. $G=\left\{G_{i j}\right\}$, and for the spatial region it occupies. We refer to cells of the virtual grid $G$ as vcells. A cell is treated as the finest element of space granularity and each spatial region can be defined by specifying the set of cells it occupies. A region $R$ cannot occupy only part of a cell: either it occupies the whole cell or it does not occupy the cell at all. We will use vcell $\sin (R)$ to denote the number of cells $R$ occupies.

The pdf $f_{\ell}(x, y)$ for any location $\ell$ is first viewed as a histogram: for each cell $G_{i j}$ the probability $p_{i j}^{\ell}$ of $\ell$ to be inside this cell is computed as $p_{i j}^{\ell}=\int_{G_{i j}} f_{\ell}(x, y) d x d y$. For an event with location $\ell$, we are naturally interested in the set of all the cells in which this event can be located: $U_{\ell}=$ $\left\{G_{i j}: p_{i j}^{\ell} \neq 0\right\}$. We will call all cells for which $p_{i j}^{\ell}=0$ the zero-cells (for $\ell$ ). Since $f_{\ell}(x, y)$ is a pdf, $\sum_{G_{i j} \in U_{\ell}} p_{i j}^{\ell}=1$. We will use the notation $U_{\ell}$ to refer to both the set of cells as well as the region they occupy. Notice that the latter simply defines an uncertainty region for $\ell$. The uncertainty region $U_{\ell}$ along with $p_{i j}^{\ell}$ for each $G_{i j} \in U_{\ell}$ defines the histogram $H_{\ell}$ for $f_{\ell}(x, y): H_{\ell}=\left\{U_{\ell},\left\{p_{i j}^{\ell}: G_{i j} \in U_{\ell}\right\}\right\}$.

At this point a naïve solution is to represent (and store on disk) each pdf as a histogram, e.g. by first identifying the minimum bounding rectangle (MBR) for the histogram and

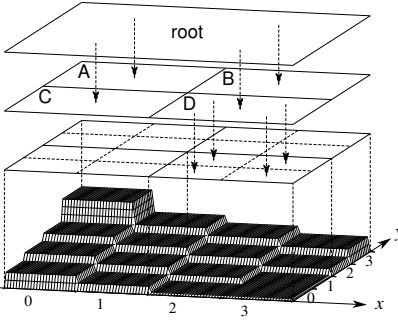

(a) Before compression.

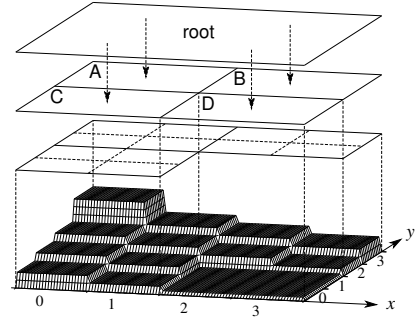

(b) After compression.
Figure 2: Quad-tree representation of pdf.

then treating the cells inside the MBR as a 2-dimensional array of real values, which can be stored sequentially on disk.

By replacing a pdf $f_{\ell}(x, y)$ with its histogram $H_{\ell}$ we essentially quantize this pdf: we approximate $f_{\ell}(x, y)$ with a different pdf $f_{H_{\ell}}(x, y)$. The new pdf $f_{H_{\ell}}(x, y)$ has constant value inside each cell $G_{i j}: f_{H_{\ell}}(x, y)=\frac{1}{\delta^{2}} \int_{G_{i j}} f_{\ell}(x, y) d x d y=$

$\frac{p_{i j}^{\ell}}{\delta^{2}}$ for $(x, y) \in G_{i j}$. Also, the histogram $H_{\ell}$ can be viewed as the probability mass function (pmf) that corresponds to $f_{\ell}(x, y)$ given that the domain $\Omega$ is viewed not as a continuous space but as a discrete space of finite number of cells.

When we approximate a pdf with a histogram representation, we lose the information about the precise shape of the pdf, but, in return, we gain several advantages. The main advantage is that manipulations with pmfs are less computationally expensive than the corresponding operations with pdfs, which involve costly integrations. This is essential, given that many types of applications require quick query response time.

It is straightforward to show that all the formulas described in the previous section, which involve pdfs and integration, can now be reformulated in terms of summations and probabilities $p_{i j}^{\ell}$ 's. E.g., in Defn. 1, the formula $\mathrm{P}(\ell \in$ $R)=\int_{R} f_{\ell}(x, y) d x d y$ becomes $\mathrm{P}(\ell \in R)=\sum_{i j: G_{i j} \in R} p_{i j}^{\ell}$.

\subsection{Quad-tree representation of pdfs}

We might be able to improve the histogram representations of pdfs further by making two observations.

Firstly, a histogram as a representation of pdf, may require large storage overhead for the pdfs that cover large areas, especially if $G$ is a very fine grid. To reduce the storage overhead as well as the number of I/Os required to retrieve a pdf from disk (thus improving the execution time), the analyst must be able to specify that any representation of a given pdf must fit in $s_{\tau} \in \mathbb{N}$ amount of space. E.g., the analyst might decide that certain types of locations are just not important, and representing each finest detail of them is not required. Let us note that the text of all the original reports is stored in the database as well. Therefore, if the analyst decides to represent certain location in more detail at a later time, that can be achieved from the stored reports.

Secondly, keeping certain aggregate information about probabilities in a given histogram, may allow for more efficient query processing. For instance, consider a $\tau$-RQ with a threshold $p_{\tau}$ and a range $R$, whose overlap with the MBR of a given histogram $H_{\ell}$ for a location $\ell$ consists of only $n$ cells. Assume for each histogram we keep the maximum value $p_{\text {max }}^{\ell}$ among all the $p_{i j}^{\ell}$ probabilities in the histogram. Then, we might be able to answer the $\tau$-RQ more efficiently. Specifically, if $n p_{\max }^{\ell}<p_{\tau}$, then since $\mathrm{P}(\ell \in R) \leq n p_{\max }^{\ell}<$ $p_{\tau}$, it immediately follows that $\ell$ does not belong to the 
answer set of the $\tau$-RQ, without performing costly computations and many disk I/Os. We can generalize the idea of keeping aggregate information to multiple levels of resolution, not only at the MBR level.

To address the above observations, we can index each histogram $H_{\ell}$ using a space partitioning index, such as a quadtree. First we build a complete quad-tree $\mathcal{T}_{\ell}$ for $H_{\ell}$. The algorithm we use is consistent (and thus omitted) with that for building quad-tree for images [14], where the goal is to index the pixels that belong to a particular image. In our case, a histogram plays the role of an image, and the nonzero cells the role of the pixels.

Each node $\mathcal{N}$ of the quad-tree $\mathcal{T}_{\ell}$ is adjusted to store certain aggregate information. Assume that the bounding rectangle of $\mathcal{N}$ intersects with $n$ vcells, whose $p_{i j}^{\ell}$ values are $p_{1}, p_{2}, \ldots, p_{n}$. Observe that some of those vcells might not belong to $U_{\ell}$, and thus some of the $p_{i}$ 's can have zero values. Then, if $\mathcal{N}$ is a leaf node, it stores a positive real value $p_{\text {sum }}$, and it holds that: $p_{1}=p_{2}=\cdots=p_{n}$ and $p_{\text {sum }}=\sum_{i=1}^{n} p_{i}=n p$, where $p=p_{1}$. If $\mathcal{N}$ is an internal node, it stores two values: $p_{\text {sum }}=\sum_{i=1}^{n} p_{i}$ and $p_{\max }=\max _{i=1, \ldots, n} p_{i}$, which are used for pruning (in this case, $p_{i} \neq p_{j}$ in general). Figure 2(a) shows an example of a (3-level) complete quad-tree built on a $4 \times 4$ histogram.

Compression. The complete quad-tree pdf representation does address the issue of maintaining aggregate information at different levels of resolution. It still does not address the first concern, where the analyst can specify that any representation of a given pdf should fit into a certain amount of space $s_{\tau} \in \mathbb{N}$. The quad-tree (lossy) compression algorithm resolves this issue. It operates by maintaining the 'compressible nodes' in a priority queue. A node is compressible if all of its children are leaf nodes. In Figure 2(a), the compressible nodes are $A, B, C, D$. The algorithm extracts from the priority queue the 'best' node to compress next $\mathcal{N}$ and performs a node compression operation on $\mathcal{N}$. This operation consists of removing all the children of $\mathcal{N}$ and converting $\mathcal{N}$ into a leaf node. Semantically, the effect of this operation corresponds to replacing $p_{i j}^{\ell}$ 's of all the cells, covered by the $\mathrm{BR}$ of $\mathcal{N}$, with the same value $p$, equal to the average of those $p_{i j}^{\ell}$ 's. After each node compression operation, the histogram is replaced with another, more 'crude', histogram of the original pdf. In the resulting histograms the new $p_{i j}^{\ell}$ 's still sum up to 1 . A node compression can make the parent of $\mathcal{N}$ to become a compressible node, in which case it is inserted in the priority queue. The algorithm keeps compressing nodes until the quad-tree fits into the specified amount of space $s_{\tau}$.

The key for the priority queue can be chosen in a number of ways. Recall that for any histogram $H$ there is pdf $f_{H}(x, y)$ that corresponds to it. Intuitively, we want the shape of $f_{H}(x, y)$ for the resulting histogram $H$ to resemble/approximate that of the original pdf as closely as possible. The algorithm above tries to achieve that approximation goal in a greedy fashion, by repeatedly collapsing 'best' compressible nodes. Thus, we need to decide what constitutes the best node to compress next. Graphically, the effect of compressing a node $\mathcal{N}$ with bounding rectangle $B$ corresponds to 'flattening' of the curvature of the histogram for the cells covered by $B$. Thus, we want to choose those nodes to flatten whose corresponding histogram curvature looks the flattest so that we minimally alter the shape.

Consider a compressible node $\mathcal{N}$ with bounding rectan- gle $B$ which covers $n$ cells. Suppose the probabilities in those cells are: $p_{1}, p_{2}, \ldots, p_{n}$. By compressing this node, the probabilities will become $p_{1}=\cdots=p_{n}=\mu$, where $\mu=$ $\frac{1}{n} \sum_{i=1}^{n} p_{i}$. So, one solution is to choose $\xi=\max _{i=1, \ldots, n} p_{i}-$ $\min _{i=1, \ldots, n} p_{i}$ as the priority queue key.

Figure 2(a) shows an example where four nodes are compressible: $A, B, C, D$, where node $A$ covers cells $[0,1] \times[2,4]$, node $D$ covers cells $[2,3] \times[0,1]$, etc. The cells covered by $D$ clearly have the least variance, so $D$ is picked for compression. Figure 2(b) shows the result of compressing $D$. Notice the flattening effect in the cells covered by $D$.

\section{INDEXING}

Assume the goal is to evaluate a $\tau$-RQ with some threshold. The quad-tree representation of pdfs might help to evaluate this query over each individual event location $\ell \sim$ $f_{\ell}(x, y)$ stored in the database faster. However, if nothing else is done, answering this query will first require a sequential scan over all the event locations stored in the database, which is undesirable both in terms of disk $\mathrm{I} / \mathrm{O}$ as well as CPU cost.

To solve this problem we can create a directory index on top of $U_{\ell}$ (or, MBR of the histogram) for each location $\ell$ in the database, using an index of our choice, such as $\mathrm{R}^{*}$ tree [1]. This solution is illustrated in Figure 3. When processing the $\tau$-RQ with region $R$, we can effectively use this index to prune away all the $U_{\ell}$ which do not intersect with $R$. Similar techniques have been studied in $[3,6]$. This method is known to lead to improvement when compared to a sequential scan. However, this method essentially utilizes only the spatial part of a pdf (i.e., $U_{\ell}$ or the MBR) and disregards the fact that there is another 'dimension' that can be used for pruning as well - the values of the pdf. Let us note that 'dimension' here is used figuratively, as it is not really a dimension, as defined in the traditional multidimensional indexing. Consequently, there is no straightforward way of applying the traditional approach of employing 3 -dimensional index $[6,15]$. Instead, the challenge becomes to create an indexing solution that is capable of using the values of pdfs. We next discuss how to solve this challenge.

\subsection{U-grid}

In this section, we propose a novel U-grid (Uncertain grid) indexing structure to effectively index uncertain event data. In addition to storing spatial information in the grid files $[9,10,13]$, U-grid also stores probability summarizations, which can be effectively utilized by the query processor.

Unlike the virtual grid $G$, the directory U-grid $I$ is materialized. The grid $I$ is much coarser than $G$ and can be viewed as a small 2-dimensional array of cells $I=\left\{I_{i j}\right\}$, residing in main memory (but it can also be stored on disk). As before, we will use $I_{i j}$ to refer to both: the cell and the region it represents. We will refer to cells in the directory grid $I$ as dcells. The structure of the grid $I$ is shown in Figure 4. Each cell $I_{i j}$ in the grid $I$ stores certain aggregate information about each event location $\ell \sim f_{\ell}(x, y)$ whose uncertainty region $U_{\ell}$ intersects with $I_{i j}$. Let us denote the set of those locations as the 'set' $L_{i j}$.

The most important information in $I_{i j}$ is a pointer to a disk-resident list, called the 'list' $L_{i j}$. For each event location $\ell$ in the set $L_{i j}$, there is a list-element $e_{\ell}$ in the list $L_{i j}$, which stores aggregate information $\left\{\right.$ oid, $\left.p_{\max }, p_{\text {sum }}, M B R\right\}$ for $\ell$, as illustrated in Figure 4 . The attribute $e_{\ell}$.oid points 


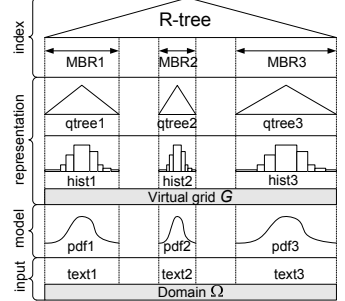

Figure 3: R-tree.

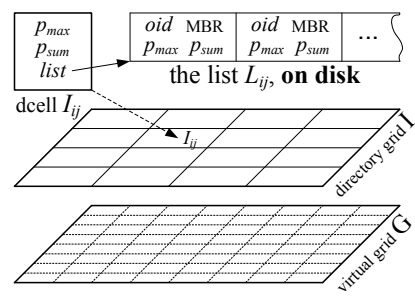

Figure 4: Grid.

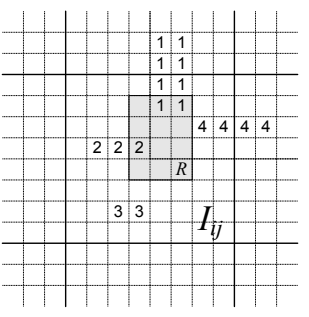

Figure 5: $\tau$-RQ in $I_{i j}$

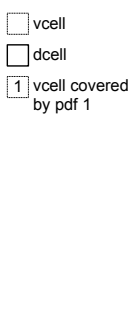

Figure 6: RQ: $I_{i 1}, I_{i 2}$ to the location of the quad-tree for $\ell$, on disk. The MBR of $\ell$ is stored in $e_{\ell} . M B R$. Let $n=\operatorname{vcell} \sin \left(U_{\ell} \cap I_{i j}\right)$, and let $p_{1}, p_{2}, \ldots, p_{n}$ be the values of the $p_{i j}^{\ell}$ probabilities of the $n$ vcells in $U_{\ell} \cap I_{i j}$. Then, $e_{\ell} \cdot p_{\max }$ and $e_{\ell} . p_{\text {sum }}$ store the values: $e_{\ell} \cdot p_{\max }=\max _{i=1, \ldots, n} p_{i}$ and $e_{\ell} \cdot p_{\text {sum }}=\sum_{i=1}^{n} p_{i}$. The list is sorted in descending order on either $p_{\text {sum }}$ or $p_{\max }$.

Clustering. Observe that we also can retrieve $\left\{p_{\max }\right.$, $\left.p_{\text {sum }}, M B R\right\}$ information directly from the root node of the quad-tree for $\ell$, instead of the list-element $e_{\ell}$ for $\ell$, so why do we store it in $e_{\ell}$ again? The reason is that, when we analyze elements of the list sequentially, if we access the quad-trees, we will incur an extra disk I/O per each location stored in the list. By storing all those attributes we achieve clustering of locations.

Other aggregate information. The lists in the directory grid $I$ can store other aggregate information for the purpose of pruning, not only the information from the root node of the quad tree $\left\{p_{\max }, p_{\text {sum }}, M B R\right\}$. We refer to this technique as L0Sketch (level-0 sketch).

We can naturally extend this technique, by storing more levels of the quad-tree. In particular, we shall see that using L1Sketch produces the best results. L1Sketch requires small additional overhead, compared to L0Sketch, since children's Bounding Rectangles (BR) can be derived from $e_{\ell} . M B R$, and thus they do not need to be stored. The pruning power of L1Sketch outweighs that of L0Sketch and easily compensates for the storage overhead.

One can also consider storing $x$-bounds here, but that turned out not to work well in practice.

Other attributes of $I_{i j}$. In addition to the pointer to the list $L_{i j}$, the dcell $I_{i j}$ also has $p_{\max }$ and $p_{\text {sum }}$ attributes, which store the maximum over all $p_{\text {max }}$ 's and over all $p_{\text {sum's }}$ 's in the list $L_{i j}$ respectively. In the next section, we discuss methods for efficient query processing using the grid.

\section{EFFICIENT QUERY PROCESSING}

Processing of $\tau$-RQs and $\tau$-PRQs consists of two logical phases: the index (pruning) phase and the object (postprocessing) phase. The first phase employs the directoryindex to prune the locations that cannot satisfy the query. Two different factors are important in this process: the speed and the quality of pruning. The locations that cannot be pruned using the directory index are inserted in the special list $L_{\text {proc }}$ to be post-processed later, on the second phase. The way in which each individual event location $\ell \in L_{\text {proc }}$ is post-processed, with respect to a given query, is independent from the choice of directory-index. The procedure that achieves that is rather straightforward. It simply traverses the quad-tree to compute the desired probability for a given query. It might stop earlier, without computing the total probability, e.g. when it becomes clear that the probability for given $\ell$ to satisfy the query either will or will not exceed the threshold $p_{\tau}$. The details of that procedure are omitted.

\subsection{Processing $\tau$-RQs using U-grid}

In this section, we present the algorithms for processing of a $\tau$-RQ with a range $R$ and a threshold $p_{\tau}$ using the directory $\mathrm{U}$-grid index $I$. The idea of the solution is to avoid the costly computation of the exact probability for a location $\ell$ to be in $R$, i.e. $\mathrm{P}(\ell \in R) \stackrel{\text { def }}{=} \sum_{i j: G_{i j} \in U_{\ell} \cap R} p_{i j}^{\ell}$. We accomplish that by being able to find a good upper-bound $\lambda$ for it, such that $\mathrm{P}(\ell \in R) \leq \lambda$. The value $\lambda$ will be computed based on the aggregate information stored in the grid $I$. The pruning will utilize the fact that, if $\lambda<p_{\tau}$, then $\mathrm{P}(\ell \in R) \leq \lambda<p_{\tau}$, and thus $\ell$ cannot satisfy the $\tau$-RQ and can be pruned without computing the exact value of $\mathrm{P}(\ell \in R)$.

One-cell scenario. Let us first consider the case where $R$ is completely inside of only one cell $I_{i j}$ of $I$, as illustrated in Figure 5. The pruning phase of the $\tau$-RQ algorithm consists of four levels of pruning:

(1) The grid-level pruning. Given $R$ intersects with only one dcell $I_{i j}$, we know that the grid index $I$ is constructed such that only the locations in $L_{i j}$ can satisfy the $\tau$-RQ. Thus, the other locations can be pruned.

(2) The dcell-level pruning. Let us set $\lambda=\min (m \times$ $\left.I_{i j} . p_{\text {max }}, p_{\text {sum }}\right)$, where $m=\operatorname{vcell} \sin (R)$. Observe that if $\lambda<p_{\tau}$, then no object $\ell \in L_{i j}$ satisfies the $\tau$-RQ, because $\mathrm{P}(\ell \in R) \leq \lambda<p_{\tau}$. Let us note that this pruning is carried out using only the content of the dcell $I_{i j}$, without performing any disk I/Os.

(3) The list-level pruning. If the above condition does not hold, the algorithm starts to process the disk-resident list $L_{i j}$ sequentially. In the example shown in Figure 5 , the list $L_{i j}$ will consist of four elements, for the locations $\ell_{1}, \ell_{2}, \ell_{3}, \ell_{4}$. Assume that the elements in $L_{i j}$ are sorted in descending order of $p_{\text {sum }}$. Suppose that the algorithm currently observes a list-element $e_{\ell}$ that corresponds to a location $\ell$. Let us choose $\lambda=e_{\ell} \cdot p_{\text {sum }}$. Observe that if $\lambda<p_{\tau}$, then neither $\ell$ nor the rest of the location in the list will satisfy the $\tau$-RQ, i.e. all of them can be pruned.

(4) The element-level pruning. If the above pruning is not successful for the element $e_{\ell}$, the algorithm might still be able to prune $\ell$ alone, but not the whole list. Let us set $\lambda=n \times e_{\ell} \cdot p_{\max }$, where $n=\operatorname{vcell} \sin \left(R \cap e_{\ell} . M B R\right)$. If $\lambda<p_{\tau}$, then $\ell$ is pruned. Else, $\ell$ is not pruned, and $\ell$ is inserted in the list $L_{\text {proc }}$ to be post-processed. After that, the algorithm extracts the next element $e_{\ell} \in L_{i j}$ and goes to Step 3 to apply the list-level pruning until all the element of $L_{i j}$ are processed. We can use L1Sketch to further reduce the upper bound by summing up the estimations from each quadrant: $\lambda=\sum_{i=1,4} n_{i} \times e_{\ell} \cdot p_{\max \_}$.

Two-cell scenario. Let us now consider the situation 


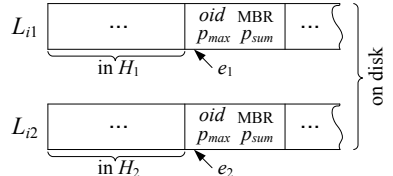

Figure 7: Two cells

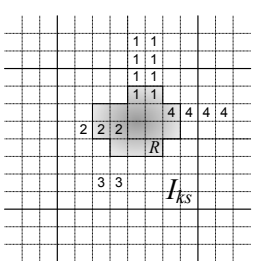

Figure 8: PRQ in $I_{k s}$

\begin{tabular}{|l|c|cc|ccc|}
\hline Strategies & Novel & \multicolumn{2}{|c|}{$\mathbf{R}^{*}$-tree } & \multicolumn{3}{c|}{ U-grid } \\
& & internal & leaf & dcell & list & element \\
\hline (1) Spatial-bound & & $\mathrm{Y}$ & $\mathrm{Y}$ & $\mathrm{Y}$ & $\mathrm{Y}$ & $\mathrm{Y}$ \\
(2) x-bound & & $\mathrm{Y}$ & $\mathrm{Y}$ & & & $\mathrm{Y}$ \\
\hline (3) Max-Sum (MS) & $\mathrm{Y}$ & $\mathrm{Y}$ & $\mathrm{Y}$ & $\mathrm{Y}$ & $\mathrm{Y}$ & $\mathrm{Y}$ \\
(4) L1 Sketch (L1) & $\mathrm{Y}$ & & $\mathrm{Y}$ & & & $\mathrm{Y}$ \\
(5) CS & $\mathrm{Y}$ & & $\mathrm{Y}$ & $\mathrm{Y}$ & $\mathrm{Y}$ & $\mathrm{Y}$ \\
\hline
\end{tabular}

Table 1: Pruning Strategies

preference region $R(x, y)$ can be viewed as the set of tuples: $\left\{\left(G_{i j}, r_{i j}\right): r_{i j} \neq 0\right\}$ and Equation 1 transforms into $\mathrm{P}(\ell \in$

\section{EXPERIMENTAL EVALUATION}

In this section, we experimentally study the efficiency of the proposed approach. We ran all our experiments on a P4-2GHz PC with 1GB RAM. The results will demonstrate the significant speedup achieved by the proposed solution, compared to the current state of the art methods.

\subsection{Experimental Setup}

Indexing methods. Table 1 summarizes the basic probability summarization techniques that can be used by an indexing structure. The existing techniques are 1 and 2, see [15]. The new ones, proposed in this paper, are 3, 4, and 5 . While the new techniques have been described in the context of U-grid, one can notice that some of them are also applicable to $\mathrm{R}^{*}$-tree and vice versa. Thus, to have a complete study, we also incorporate our techniques in $\mathrm{R}^{*}$-tree and evaluate their results as well.

Table 1 illustrated to which part of various indexing structures $\left(\mathrm{R}^{*}\right.$-tree and $\mathrm{U}$-grid) a given summarization technique is applicable. For instance, it shows that L1 technique can be applied to the element level of U-grid and leaf level of

Assume a $\tau$-PRQ has a threshold $p_{\tau}$ and a preference
egion $R(x, y)$. Let us denote the average value of $R(x, y)$
in the cell $G_{i j}$ as $r_{i j}: r_{i j}=\frac{1}{\delta^{2}} \int_{G_{i j}} R(x, y) d x d y$. Then,

Assume a $\tau$-PRQ has a threshold $p_{\tau}$ and a preference
region $R(x, y)$. Let us denote the average value of $R(x, y)$
in the cell $G_{i j}$ as $r_{i j}$ : $r_{i j}=\frac{1}{\delta^{2}} \int_{G_{i j}} R(x, y) d x d y$. Then,

Assume a $\tau$-PRQ has a threshold $p_{\tau}$ and a preference
region $R(x, y)$. Let us denote the average value of $R(x, y)$
in the cell $G_{i j}$ as $r_{i j}$ : $r_{i j}=\frac{1}{\delta^{2}} \int_{G_{i j}} R(x, y) d x d y$. Then,

\begin{abstract}
$R) \stackrel{\text { def }}{=} \sum_{i j: G_{i j} \in R \cap U_{\ell}} p_{i j}^{\ell} r_{i j}$. The goal is, to avoid the costly computation of the exact probability $\mathrm{P}(\ell \in R)$ by being able to find a good upper-bound $\lambda$ for it, such that $\mathrm{P}(\ell \in$ $R) \leq \lambda$. The value of $\lambda$ will be derived from the aggregate information stored in the directory grid $I$. The pruning will be based on the observation that, if $\lambda<p_{\tau}$, then we can prune $\ell$ since it cannot be in the answer set of the $\tau$-PRQ.

Due to the page limit, we study only the scenario illustrated in Figure 8, where $R$ intersects with only one dcell $I_{k s}$. The technique generalizes to any number of cells. First observe that any preference region $R(x, y)$ induces a regular region $R^{\prime}(x, y)=\{(x, y): R(x, y) \neq 0\}$. That regular region can be obtained by treating all non-zero $r_{i j}$ 's of the preference region as 1's. Observe that $\mathrm{P}(\ell \in R)=$ $\sum_{i j: G_{i j} \in R \cap U_{\ell}} p_{i j}^{\ell} \times r_{i j} \leq \sum_{i j: G_{i j} \in R \cap U_{\ell}} p_{i j}^{\ell} \times 1=\mathrm{P}\left(\ell \in R^{\prime}\right)$. Thus, we can use the techniques from Section 6.1 to upperbound $\mathrm{P}\left(\ell \in R^{\prime}\right)$ and that would upper-bound $\mathrm{P}(\ell \in R)$ as well, because $\mathrm{P}(\ell \in R) \leq \mathrm{P}\left(\ell \in R^{\prime}\right) \leq \lambda<p_{\tau}$.

Cauchy-Shwarz inequality. The most effective technique employed for pruning for $\tau$-PRQ is based on CauchyShwarz inequality: $\sum_{i=1}^{n} x_{i} y_{i} \leq\left(\sum_{i=1}^{n} x_{i}^{2}\right)^{\frac{1}{2}}\left(\sum_{i=1}^{n} y_{i}^{2}\right)^{\frac{1}{2}}$. Using it, we have: $\mathrm{P}(\ell \in R) \stackrel{\text { def }}{=} \sum \ldots \leq\left(\sum_{i j: G_{i j} \in I_{k s}}\left(p_{i j}^{\ell}\right)^{2}\right)^{\frac{1}{2}} \times$ $\left(\sum_{i j: G_{i j} \in I_{k s}}\left(r_{i j}\right)^{2}\right)^{\frac{1}{2}}=S_{\ell} S_{R}$, where $S_{\ell}$ and $S_{R}$ are the two $(\cdot)^{\frac{1}{2}}$ terms. The value $S_{R} \in \mathbb{R}$ is computed once per query $R$ and cell $I_{k s}$. The value $S_{\ell} \in \mathbb{R}$ is apriori stored in each element $e_{\ell}$ of the list $L_{k s}$ along with other aggregate information. Now, when processing list-element $e_{\ell} \in L_{i j}$, we can use $\lambda=S_{R} \times e_{\ell} . S_{\ell}$ as one of the upper-bounds for $\mathrm{P}(\ell \in R)$.

one dcell. We study only two-cell scenario for illustration purposes; the technique generalizes to any number of dcells.
(1) The grid-level pruning. Assume that $R$ intersects with only two dcells: $I_{i 1}$ and $I_{i 2}$. We know that the index grid can satisfy the $\tau$-RQ, the rest of the locations are pruned. The dcell-level pruning. Let $m_{1}=$ vcellsin $\left(R \cap I_{i 1}\right)$ $\min \left(m_{2} \times I_{i 2} . p_{\max }, I_{i 2} . p_{\text {sum }}\right)$. Then, if $\lambda<p_{\tau}$, then no objects in $L_{i j}$ satisfies the $\tau$-RQ, hence all of them are pruner two lists $L_{i 1}$ and $L_{i 2}$ at the same time in a sequential manthose lists are sorted in descending order of $p_{\text {sum. }}$. Suppose that the algorithm currently observes elements $e_{1} \in L_{i 1}$ and note that $\ell_{1}$ and $\ell_{2}$ can be the same object. All the pre-
\end{abstract} we have already observed $\ell_{1}$ on the previous steps. Namely, there is already an element $e_{\ell_{1}}$ in $\mathcal{H}_{2}$ for $\ell_{1}$, then $\lambda_{2}=$ $\lambda_{1}+\lambda_{2}$ and if $\lambda<p_{\tau}$, then we can prune $\ell_{1}$. In the latter If the above methods cannot prune $\ell_{1}$, then $\ell_{1}$ is in

After $\ell_{2}$ is processed in a similar manner, the algorithm

\subsection{Processing $\tau$-PRQs using U-grid}


$\mathrm{R}^{*}$-tree. Max-Sum (MS) refers to using $\left(p_{\max }, p_{\text {sum }}\right)$ summary to prune objects. It can apply to $\mathrm{R}^{*}$-tree and U-grid at all levels. $\boldsymbol{C S}$ is the Cauchy-Shwarz technique employed by PRQ queries. Therefore, we have many individual index variations and we will only test most prominent solutions.

To evaluate these techniques effectively, we group them under two schemes: $\mathrm{R}^{*}$-tree and U-grid. We use $\mathrm{R}^{*}$-tree with Spatial-bound pruning as our comparison baseline, to evaluate the following existing and novel techniques:

- Existing: (1) $\mathrm{R}^{*}$-tree with $x$-bounds, also known as UPCR [15], (2) $\mathrm{R}^{*}$-tree with compressed $x$-bounds, the current state of the art technique also known as U-tree [15] (3) Naïve Grid;

- Novel: (1) U-grid-MS with $M S$ pruning, (2) U-grid with $x$-bounds (U-grid-x) and with compressed $x$-bounds (U-grid- ${ }^{+}$), (3) U-grid-MSL1 for $M S$ pruning and

L1Sketch, (4) RTree-MS, (5) RTree-MSL1.

Naïve Grid is a standard grid, which does not use probabilities for pruning. Note that to reduce the indexing overhead, $x$-bounds can also be compressed using linear approximation method [15]. U-grid-x and U-PCR store nine $x$ bounds for values from 0.1 to 0.9 at the interval of 0.1 ; the compressed versions - U-grid- $\mathrm{x}^{+}$and U-tree - are built on top of these nine $x$-bounds.

Domain. We propose U-grid as the best solution for citylevel domains. In this paper, we use Manhattan Area of New York with a $400 \times 400$ virtual grid is overlaid on top of it.

Uncertain location data for testing the Efficiency is derived from reports filed by NYPD Officers. From these reports, 2359 uncertain locations are extracted and mapped into the probabilistic representation, using the framework from [8]. The number of the locations might not be sufficient for testing the scalability. Hence, we have generated several larger $(10 \mathrm{~K}-100 \mathrm{~K})$ synthetic datasets, based on this real dataset, as follows. We first partition the real events into three uncertainty categories, based on the size of their spatial uncertainty regions: the low (the pdf covers less than $10 \times 10$ vcells), medium (less than $20 \times 20$ vcells), and high (less than $100 \times 100$ vcells). To generate a synthetic location, we randomly choose a real uncertain location, and generate a new one with a similar pdf on top of a landmark (building, street intersection) in the domain. When storing the new pdf as a quad-tree and paginating it to the disk, an average-sized pdf occupies 5 to 10 disk pages. Using the lossy compression techniques, we constrain the size of any pdf to 100 pages maximum.

We can now control the data uncertainty level of a synthetic dataset, by mixing objects with different uncertainty levels. In our experiments, a dataset with the medium data uncertainty level has object uncertainty mixture ratio of $\left(l_{0} w^{90 \%}:\right.$ med $^{5 \%}$ : high $\left.^{5 \%}\right)$, the low and high levels are defined as: low $=(98 \%: 1 \%: 1 \%)$, and high $=(50 \%:$ $30 \%: 20 \%$ ). The medium data uncertainty has roughly the same mixture ratio as the 2359 real events.

Queries. Similar to the concept of object uncertainty levels, spatial queries have the low, medium and high coverage levels to characterize query size: $10 \times 10,20 \times 20$, and $100 \times 100$ vcells. They also have query uncertainty levels to characterize mixes of queries of various coverage levels: low / med / high are the same as data uncertainty levels.

Table 2 summarizes the default experimental settings. We vary those settings to analyze the performance of the different indexing strategies. For each setting, we execute a large

\begin{tabular}{|l|c|}
\hline Parameter & Value \\
\hline Data Size & 30,000 \\
$p_{\tau}$ & 0.8 \\
Grid Size & $16 \times 16$ \\
\hline
\end{tabular}$\quad$\begin{tabular}{|l|c|}
\hline Parameter & Value \\
\hline Data Uncertainty & Medium \\
Query Uncertainty & Medium \\
Page Size & 1024 \\
\hline
\end{tabular}

Table 2: Default System Settings

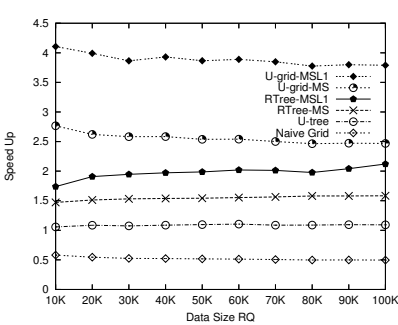

Figure 9: Data Size

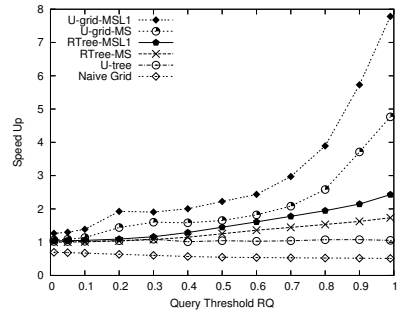

Figure 10: Threshold number of spatial queries and then report the average disk $\mathrm{I} / \mathrm{O}$ per one query.

\subsection{Experiments}

Table 3 studies the impact of different database sizes and query thresholds on the I/O cost of $\tau$-RQs for the different indexing techniques. All tested techniques are several orders of magnitude better than linear scan (not shown). In terms of the overall execution time, linear scan takes more than 2 minutes to complete even for $10 \mathrm{~K}$ of data. All the techniques, except for linear scan, have two phases as discussed in the beginning of Section 6: the indexing (phase I) and post-processing (phase II).

Data size. Table 3 shows the phase I and total I/O costs separately. U-grid-MS has the best phase I costs, and Ugrid-MSL1 has the best overall costs. In all experiments, Rtree-MS has better performance than R-tree (baseline). This proves $M S$ pruning is effective in R-tree index. However, the existing techniques based on the $\mathrm{x}$-bounds (U-PCR and U-tree) do not perform well. This shows that the $x$ bound idea does not work well in 2-d case; the overhead in storing the $\mathrm{x}$-bounds and compressed $\mathrm{x}$-bounds clearly outweighs the gains from probability pruning. For a U-PCR indexing node, it needs to store additional 36 real numbers than a R-tree node. This reduces fan out by more than 8 times. Even with the compression techniques, to store the parameters used in the linear approximations, U-tree still needs to reduce the fan out by more than 4 times. The Utree's performance is only slightly better than that of the baseline, and U-PCR has the worst overall performance.

Figure 9 shows the improvement ratio (speedup) of various indexing techniques, compared to the baseline (R-tree). The best technique from Grid group is U-grid-MSL1. It shows 4 times speedup over the baseline, and also almost 4 times improvement over the best existing solution, U-tree.

The best technique from R-tree group is Rtree-MSL1, but it is only about 2 times better than the baseline. The main reason for this difference is that for U-grid, the regions that correspond to lists are dcells, hence they cannot overlap; but, for RTree-List, the regions can (and do) overlap. Hence, the probabilistic pruning techniques become less effective. The experimental results also show that L1Sketch is a very effective technique for both, R-tree and U-grid.

Query Threshold. Table 3 also shows similar behavior when the query threshold $p_{\tau}$ for $\tau$-RQs is varied. As we can observe from Figure 10, the gain ratio for U-grid-MSL1 gets 


\begin{tabular}{|c|c|c|c|c|c|c|c|c|c|c|c|c|c|}
\hline \multirow{3}{*}{ Methods } & \multirow{3}{*}{ Novel } & \multicolumn{6}{|c|}{ Overall I/O Cost } & \multicolumn{6}{|c|}{ PhaseI I/O Cost } \\
\hline & & & Size & & & $\tau$ & & & Size- & & & $\tau$-I & \\
\hline & & 10k & $50 \mathrm{k}$ & 100k & 0.1 & 0.5 & 0.8 & $10 \mathrm{k}$ & $50 \mathrm{k}$ & 100k & 0.1 & 0.5 & 0.8 \\
\hline$\overline{\mathrm{R}^{*} \text {-tree }}$ & & 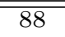 & 385 & $\overline{7740}$ & 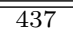 & 259 & 235 & $\overline{24}$ & 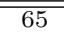 & $\overline{\overline{98}}$ & 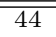 & 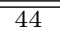 & $\overline{444}$ \\
\hline U-PCR & & 109 & 457 & 853 & 553 & 306 & 238 & 60 & 209 & 359 & 160 & 136 & 136 \\
\hline U-tree & & 83 & 351 & 677 & 475 & 241 & 219 & 34 & 104 & 183 & 82 & 72 & 72 \\
\hline Rtree-MS & $\mathbf{Y}$ & 60 & 250 & 467 & 432 & 207 & 154 & 22 & 60 & 95 & 40 & 39 & 37 \\
\hline U-tree-MS & $\mathbf{Y}$ & 68 & 279 & 520 & 474 & 221 & 180 & 34 & 102 & 168 & 87 & 76 & 76 \\
\hline Rtree-MSL1 & $\mathbf{Y}$ & 51 & 194 & 349 & 414 & 178 & 121 & 36 & 116 & 194 & 73 & 68 & 60 \\
\hline Naïve Grid & & 152 & 746 & 1489 & 650 & 472 & 449 & 6 & 23 & 43 & 14 & 14 & 14 \\
\hline U-grid-MS & $\mathbf{Y}$ & 32 & 152 & 300 & 384 & 157 & 91 & $5 *$ & $16 *$ & $29 *$ & $14^{*}$ & $12^{*}$ & $10 *$ \\
\hline U-grid- $x^{+}-M S$ & $\mathbf{Y}$ & 37 & 174 & 343 & 407 & 162 & 104 & 11 & 43 & 83 & 38 & 31 & 27 \\
\hline U-grid-MSL1 & $\mathbf{Y}$ & $22^{*}$ & $100 *$ & $195 *$ & $315 *$ & $116^{*}$ & $60 *$ & 10 & 39 & 74 & 34 & 27 & 25 \\
\hline
\end{tabular}

Table 3: Disk I/O for Various Index Strategies

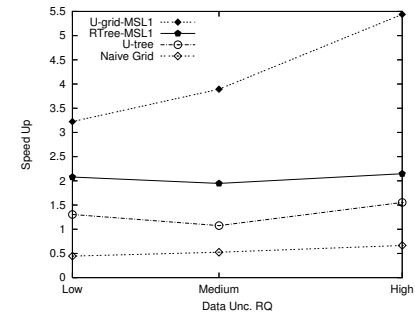

Figure 11: Data Unc.

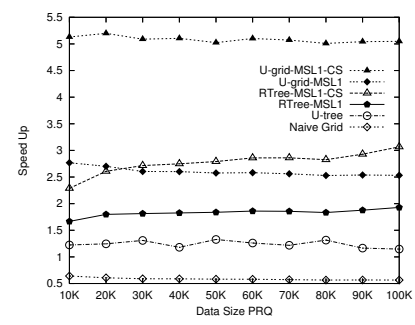

Figure 13: PRQ

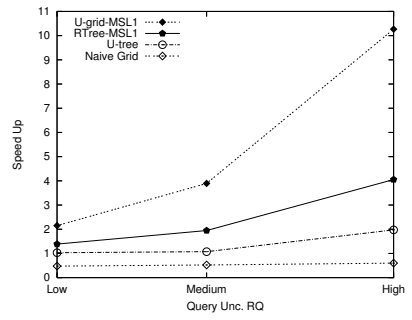

Figure 12: Query Unc.

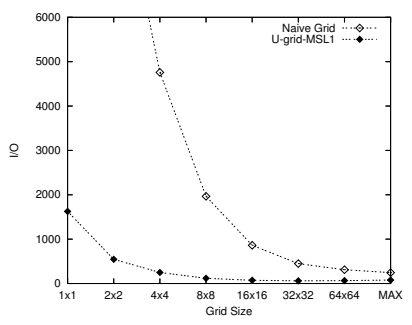

Figure 14: Grid size even better (up to the factor of 8) when the threshold increases. It shows the combined pruning strategy (MS+L1) can dismiss most of the false positive objects instead of pushing them to phase-II.

Misc. performance. In Figures 11 and 12 we vary the data and query uncertainty levels. As data and query become more uncertain, pruning in phase I becomes very important since there is more objects intersecting with the query region. The results show that our combined pruning strategy $(\mathrm{MS}+\mathrm{L} 1)$ works very well for U-grid, but less effective for R-tree, since the overlapping MBRs in R-tree degrade its performance.

U-grid size. Figure 14 plots the impact of the grid size on the performance of $\tau$-RQs for the U-grid-MSL1 technique. When the U-grid has only small number of cells, e.g. $1 \times 1$ or $2 \times 2$ cells, the overall I/O cost is high, since many objects are pushed to phase-II for post-processing. As the number of cells increases, the I/O cost stabilizes: having finer than $16 \times 16$ grid does not lead to significant improvement. Therefore, keeping the directory grid $I$ in main memory, except for its disk-resident $L_{i j}$ lists, is feasible.

PRQs. Figure 13 studies the effect of the data size on the performance of $\tau$-PRQs. The new technique, U-grid-MSL1CS, achieves 5 time speedup over the baseline, and 4 time speedup over the best existing solution, U-tree.

\section{CONCLUSION}

This paper presents a solution for indexing and fast retrieval of uncertain spatial point data, reflected as pdfs. The proposed approach will benefit a variety of applications that require fast query processing over uncertain (spatial) data. The proposed grid-based index, U-grid, achieves its effectiveness by using values of pdfs as another indexing 'dimension', namely by employing high-level summaries/sketches of the underlying pdfs. The paper also demonstrates that (regardless of the actual underlying representation of pdfs) there can be a merit of building an individual index (e.g., Quad-tree) on top of each pdf, to summarize it, and further increase query processing speed.

\section{REFERENCES}

[1] N. Beckmann, H. Kriegel, R. Schneider, and B. Seeger. The $\mathrm{R}^{*}$-tree: An efficient and robust access method for points and rectangles. In $A C M S I G M O D, 1990$.

[2] R. Cheng, D. V. Kalashnikov, and S. Prabhakar. Evaluating probabilistic queries over imprecise data. In SIGMOD, 2003.

[3] R. Cheng, D. V. Kalashnikov, and S. Prabhakar. Querying imprecise data in moving object environments. IEEE TKDE, 16(9), Sept. 2004

[4] R. Cheng, D. V. Kalashnikov, and S. Prabhakar. Evaluation of probabilistic queries over imprecise data in constantly-evolving environments. Information Systems Journal, 2006. to appear.

[5] R. Cheng, S. Prabhakar, and D. V. Kalashnikov. Querying imprecise data in moving object environments. In ICDE, 2003.

[6] R. Cheng, Y. Xia, S. Prabhakar, R. Shah, and J. S. Vitter. Efficient indexing methods for probabilistic threshold queries over uncertain data. In Proc. of $V L D B, 2004$

[7] D. V. Kalashnikov, Y. Ma, S. Mehrotra, and R. Hariharan. Modeling and querying uncertain spatial information for situational awareness applications. In Proc. of ACM GIS, 2006.

[8] D. V. Kalashnikov, Y. Ma, S. Mehrotra, R. Hariharan, N. Venkatasubramanian, and N. Ashish. SAT: Spatial Awareness from Textual input. In Proc. of EDBT, 2006.

[9] D. V. Kalashnikov, S. Prabhakar, and S. Hambrusch. Main memory evaluation of monitoring queries over moving objects. Distributed and Parallel Databases, 15(2):117-135, Mar. 2004.

[10] D. V. Kalashnikov, S. Prabhakar, S. Hambrusch, and W. Aref. Efficient evaluation of continuous range queries on moving objects. In Proc. DEXA Conference, September 2-6 2002.

[11] S. Mehrotra, C. Butts, D. V. Kalashnikov, N. Venkatasubramanian, and et el. CAMAS: A citizen awareness system for crisis mitigation. In $A C M$ SIGMOD, 2004.

[12] S. Mehrotra, C. Butts, D. V. Kalashnikov, N. Venkatasubramanian, R. Rao, G. Chockalingam, R. Eguchi, B. Adams, and C. Huyck. Project RESCUE: challenges in responding to the unexpected. SPIE, 5304:179-192, Jan. 2004.

[13] J. Nievergelt, H. Hinterberger, and Sevcik. The grid file: An adaptable, symmetric multi-key file structure. In ECI'81.

[14] H. Samet. The Design and Analysis of Spatial Data Structures. Addison-Wesley, 1990.

[15] Y. Tao, R. Cheng, X. Xiao, W. K. Ngai, B. Kao, and S. Prabhakar. Indexing multi-dimensional uncertain data with arbitrary probability density functions. In $V L D B, 2005$. 